\title{
The Prediction Method of Actual Traffic Based on ARMA and Wavelet Transform
}

\author{
Chen guobin ${ }^{1, \text { a }}$ \\ ${ }^{1}$ Rongzhi College, Chongqing Technology and Business University, Chongqing, 400033, China \\ aemail:759327776@qq.com
}

Keywords: Wireless Sensor Network; Prediction; ARMA; Wavelet; Error

\begin{abstract}
In order to improve the prediction accuracy of wireless sensor network, a novel prediction algorithm is proposed by ARMA and wavelet transform. In this algorithm, the characteristic of ARMA is defined, and the Theoretical foundation of is given. Then, the prediction error of actual traffic is decreased by fusion the results of ARMA and wavelet transform. Finally, a simulation was conducted to study the key influence factor of algorithm with OPNET and MATLAB. The results show that, compared to FARIMA model, This paper has better suitability.
\end{abstract}

\section{Introduction}

Wireless Sensor Network (Wireless Sensor Network, WSN) have unique applications, and obtained the rapid development of [1-5]. Network congestion in WSN is to reduce the performance of the key factors, in recent years, some scholars puts forward some solutions to network congestion in WSN, CODA [6], fusion [7], etc. Through the network nodes and the flow condition of congestion control. How to effectively handle before network congestion, has become a WSN important method to improve the network performance. How to control the actual flow, predict the network traffic status has been becoming the current hot and difficult. A typical network prediction method has AR, ARMA and FARIMA model. At the same time, with the deepening of the research, other methods such as wavelet transform, chaos model have also been introduced in actual traffic prediction. To this, the domestic and foreign scholars have done a lot of research work. Literature [8] with super linear convergence of the variable metric method to improve ARMA prediction model, and based on autocorrelation coefficient and partial autocorrelation coefficient trailing method of actual traffic forecasting. Literature [9] base by extending the start of the iteration and the last stage of the search time, realizes the algorithm of the balance between global search and local search ability, and optimize the model parameters, based on chaos IPSO optimization support vector machine forecasting model. Literature [10] by using the least squares support vector machine (SVM) and fuzzy LSSVM training, set up a kind of optimal sample subset online fuzzy prediction algorithm, and further study of the actual flow of degeneration and long cycles. Literature [11] in view of the traditional forecast model high dependence of training data, the combination of wavelet technology and ant colony algorithm to build the Back Propagation network weights of prediction methods, improve the prediction accuracy. Literature [12] to improve the Logistic model, based on the cosine function and using the method of nonlinear time series analysis and Logistic model to describe the evolution of the state of actual traffic situation and chaotic characteristics. Literature [13] traffic time series wavelet decomposition, the wavelet transform scale coefficients obtained sequence, and the wavelet coefficients by coefficient sequences and the flow of the original time series as input and output of the model respectively, and structure of artificial neural network to train the literature [14] for real-time update of prediction accuracy problems, set up an online fuzzy least squares support vector machine (SVM) method, but need to further consider the effect of time scale.

On the basis of the above work, this paper puts forward a new kind of actual traffic prediction algorithm, this algorithm through [fusion ARMA model and the prediction of wavelet transform, in order to reduce the prediction error, through mathematical simulation validates the effectiveness of the algorithm. At the same time the paper structure as follows: section 1 presents the characteristics 
of ARMA model, section 2 presents a judgment based on ARMA model, and to establish a prediction algorithm, section 3 experiments are carried out using OPNET and MATLAB simulation, the fourth quarter to summarize the full text.

\section{Design of the Chip Kick Mechanics the ARMA model}

Regression sliding model has high forecast precision and rapidity, timeliness is good wait for a characteristic. In the wireless sensor normal flow sequence is: $\mathrm{W} 1, \mathrm{~W} 2, \ldots \mathrm{Wt}$, the model representation is as follows:

$$
W_{t}=\sum_{i=1}^{p} \tau_{1} W_{t-i}-\sum_{j=1}^{q} \rho_{j} a_{t-j}+a_{t}
$$

Among them: $a_{t} \in N\left(0, \sigma_{a}^{2}\right) \tau_{i}, \rho_{i}$ is coefficient, $a_{t}$ is interference. Type (1) is a p order autoregressive m order moving average model and order to as ARMA (p, q); P and q respectively of AR and MA part order; $\tau_{i}(\mathrm{I}=1,2 \ldots \mathrm{n}), \rho_{i}(\mathrm{j}=1,2, \ldots, \mathrm{m})$ model parameters of each part respectively.

Wt mathematical expectation $\mathrm{E}(\mathrm{Wt})$ of the time $\mathrm{T}$ :

$$
E\left(W_{t}\right)=\sum_{i=1}^{n} \tau_{t} W_{t-i}-\sum_{j=1}^{m} \rho_{j} a_{t-j}
$$

$\mathrm{E}(\mathrm{Wt})$ affected by data Wt-I and interfere $a_{t-j}$

\section{The prediction algorithm based on ARMA model combined with wavelet}

To reach the actual flow of whether meet the prediction effect, specific inspection steps here:

$\mathrm{T}$ moment wireless sensor networks base station received the actual flow of $\mathrm{Wt}$ status to $\mathrm{Z}_{\mathrm{t}}$, here mainly aims at the time delay of the actual flow $D_{t}$ and captain Lt is analyzed, namely the $Z_{t}=\left[D_{t}\right.$, $L_{t}$ ], Lt. In order to effectively deal with the possibility of congestion phenomenon, so by the actual traffic status of the current and previous moment were analyzed, and for the next moment $\mathrm{Z}_{t+1}$ traffic status, to implement reasonable queue management. The following condition forecasting algorithm combining with the actual flow is given:

Step 1: according to the literature [15] gives the calculation method of solving $t+1$ times the business flow of time delay $\mathrm{D}_{\mathrm{t}+1}$ and captain $\mathrm{L}_{\mathrm{t}+1}$ :

$$
\begin{aligned}
D_{t+1}^{\prime} & =1-(1-\lambda) \sum_{n=0}^{\infty} \Omega_{m+n+1}((n+1) b-t) \\
L^{\prime}{ }_{t+1} & =(1-\lambda) \sum_{n=0}^{\infty} \Omega_{k+n}(n+1) b
\end{aligned}
$$

Where $\lambda$ is base load size, $\mathrm{b}$ is the buffer size, $\Omega_{\mathrm{k}+\mathrm{n}}$ is the actual traffic distribution function.

Step2: Due to the sudden and measure of actual flow characteristics, the introduction of wavelet transform algorithm to deal with traffic and prediction, and to predict the results of the step (2) the results obtained with integration, reduce the error. According to the formula (5) shown in wavelet transform, using DB2 wavelet time delay Dt to the t time the actual flow of Wt and captain Lt decomposition, to obtain the coefficient of the scale $A J(k)$ and wavelet coefficient $D J(k)$ of the $j$ layer:

$$
\begin{aligned}
& \sqrt{2} a_{j}(k)=a_{j+1}(2 k)+a_{j+1}(2 k+1) \\
& \sqrt{2} d_{j}(k)=a_{j+1}(2 k)-a_{j+1}(2 k+1)
\end{aligned}
$$

Step3: by using ARMA model to forecast the wavelet coefficients. By estimating parameters $\tau(1)$, $\tau(2), \cdots, \tau(p)$ of AR (p) and FIR filter, $A(z)=1+\sum_{k=1}^{p} \tau(k) z^{-k}$ filter, output nearly a MA (q) process: 
$\rho(1), \rho(2), \cdots, \rho(q)$ and obtained the parameters p and q, at the same time, according to the formula (6) computational prediction of wavelet coefficients, and USES the inverse wavelet transform of synthetic prediction after delay $D^{\prime \prime}{ }_{\mathrm{t}+1}$ and captain $\mathrm{q}^{\prime \prime} \mathrm{t}_{\mathrm{t}+1}$;

$$
\left\{\begin{array}{l}
D{ }_{t+1}=-\sum_{k=1}^{p} \tau_{k} D_{t-k}+\sum_{k=0}^{q} \rho_{k} u_{t-k} \\
L^{\prime \prime}{ }_{t+1}=-\sum_{k=1}^{p} \tau_{k} L_{t-k}+\sum_{k=0}^{q} \rho_{k} u_{t-k}
\end{array}\right.
$$

Step4:According to the step (2) and (4) $t+1$ point to actual traffic delay and captain state information, here has the following fusion operation, reduce the prediction error:

$$
\begin{aligned}
& D_{t+1}=\phi D_{t+1}^{\prime}+\varphi D^{\prime \prime}{ }_{t+1} \\
& L_{t+1}=\phi L_{t+1}^{\prime}+\varphi L^{\prime \prime}{ }_{t+1}
\end{aligned}
$$

Where two prediction $\varphi$ and $\phi$ is the fusion parameter results and the weight distribution, can be dynamically adjusted in order to obtain the optimal result. $0 \leq \phi \leq 1, \quad 0 \leq \varphi \leq 1, \quad \phi+\varphi=1$;

Step5: $t=t+1$, skip to step (1), double counting actual flow vector of the next moment, until the end.

Step6: Algorithm over

\section{Test results}

In order to verify the effectiveness of the algorithm, based on OPNET and MATLAB simulation experiment here. Experimental environment: Intel CPU G2020 2.9 G, 4 G memory. Firstly established in OPNET is shown in figure 1 of the wireless sensor network topology: produced 10 clusters, each cluster 5 nodes, and set up the link capacity of 4 MBPS, delay of $20 \mathrm{~ms}$, buffer capacity of 80 packets of ordinary nodes, base station buffer capacity of 600 packets, $=0.5$, wavelet decomposition $\varphi=\phi$ packet size of $128 \mathrm{~b}$, the fusion parameter level to 10 . At the same time, in order to show that the prediction precision of the algorithm and here, too, will be used for fractal characteristics depicting FARIMA model [16]. Two algorithms are used in the experimental environment simulation 30 times in a row, after take the average value, figure 2 and figure 3 shows the two algorithms respectively the prediction results of actual traffic delay and captain. Can be seen from the figure 2 and figure 3, the proposed in this paper, the flow and the flow is relatively close, while FARIMA model has a certain error. Analysis of the reason is that FARIMA model computation complex, in the practical application often delayed the true state of dynamic change, therefore resulting in big error. Comprehensive error analysis of experimental results, the algorithm and FARIMA model reached $145.34 \%$ and $21.57 \%$ respectively.

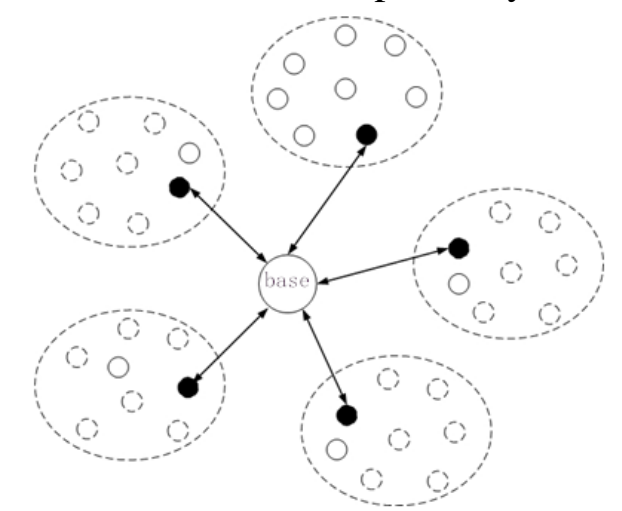

Figure 1 simulation environment 


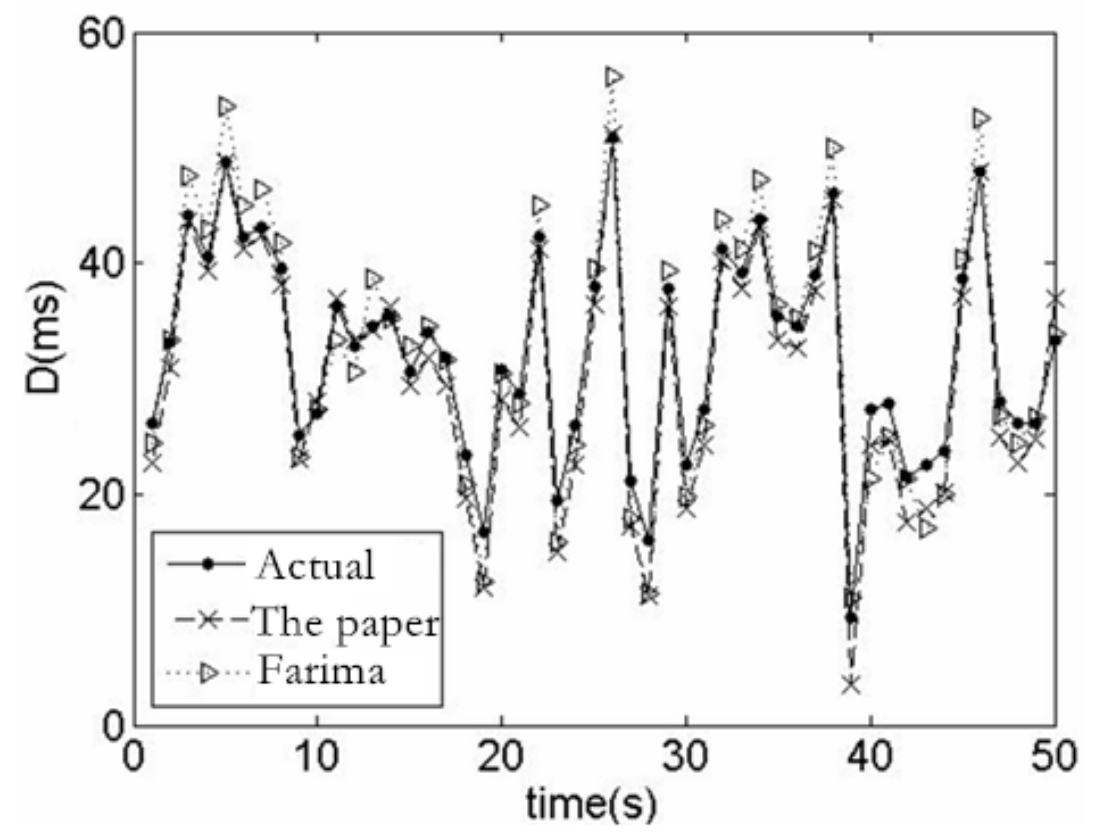

Figure 2 time delay prediction results

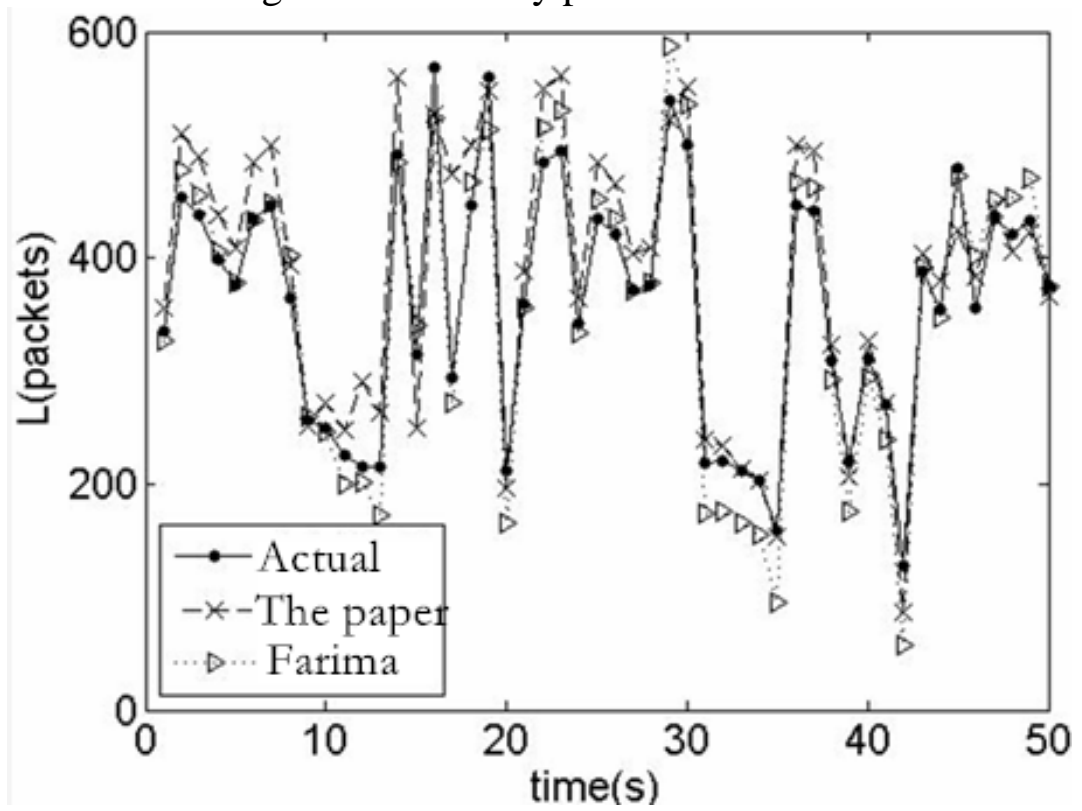

Figure 3 captain predicted results compared

Second, its in-depth study on the performance of algorithm in this paper, discuss its key parameter change on the impact of the performance. Here introduced the actual flow of $X$ and the related parameters of the characteristic function: remember, the formula (9) :

$$
\Phi(\omega)=E\left[e^{j \omega X}\right]=\left\{\begin{array}{l}
\exp \left\{j \mu \omega-|\sigma \omega|^{\alpha}\left[1-j \beta \operatorname{sgn}(\omega) \tan \left(\frac{\pi \alpha}{2}\right)\right]\right\}, \alpha \neq 1 \\
\exp \left\{j \mu \omega-|\sigma \omega|^{\alpha}\left[1+j \beta \operatorname{sgn}(\omega) \frac{2}{\pi} \ln |\omega|\right]\right\}, \alpha=1
\end{array}\right.
$$

Where: $\alpha(0<\alpha<2)$ as the characteristic parameter, used to represent the state of the emergency degree and the fractal of actual flow; $\beta(-1 \leq \beta \leq 1)$ for deflection parameters, used to represent the degree of deviation, through a and $\beta$, can determine the data flow distribution density function of the shape. $\sigma(\sigma \geq 0)$ as the scale parameter deviation, $\mu$ as the location parameters, used to represent the distribution of the degree of deviation and average. On the algorithm performance under different sudden situation, figures 4 and 5, respectively is given under different parameter $\phi$ prediction error of time delay. Can be seen from the $\phi$ the captain and changes of the fusion between parameters figure 4, with the increase of parameters $\delta$ of the delta, after the delay prediction error is presented to reduce the increasing trend. And when the parameters $\delta$ of the delta is small, the greater the $\alpha$ 
corresponding time delay prediction error is smaller, and when the parameters of the delta is larger, the greater the $\alpha$ corresponding prediction error is bigger. This is because the greater the $\alpha$ means that the actual flow of sudden, the greater the combination of ARMA model and wavelet transform algorithm used in this paper to forecast processing, parameters of the delta smaller means that wavelet transform function $(\delta+\eta=1)$, the greater the wavelet transform to eliminate the sudden has good effect, thus show the corresponding error is smaller. And parameters of the $\delta$ is larger $\alpha$ play a major role, for processing low sudden has good effect, so the $\alpha$, the smaller the corresponding prediction error is smaller. Similar phenomenon can also be found in figure 4, when the parameters of the $\delta$ is small, the greater the $\alpha$ corresponding queue prediction error is smaller, and when the parameters of the $\delta$ is bigger, the change situation.

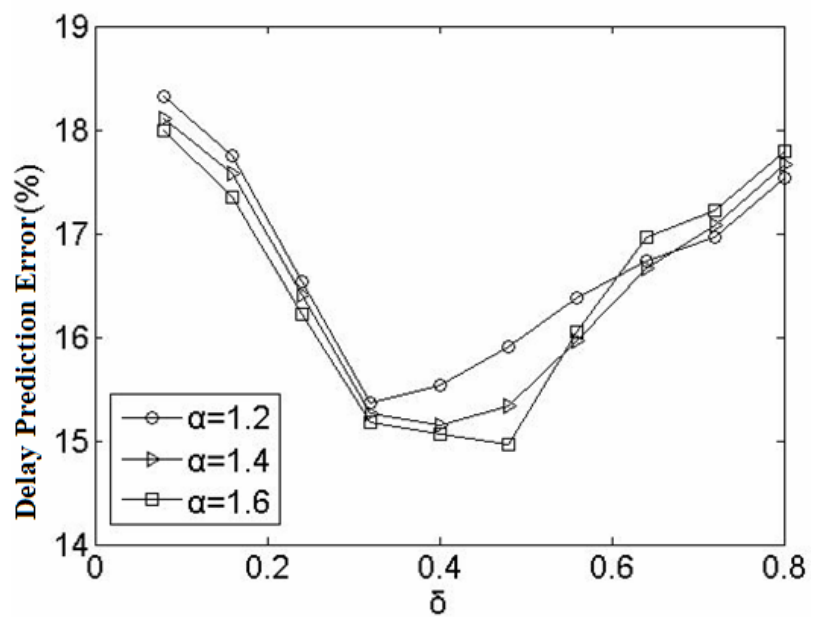

Figure 4 time delay prediction error and changes in the relations between the parameters $\delta$

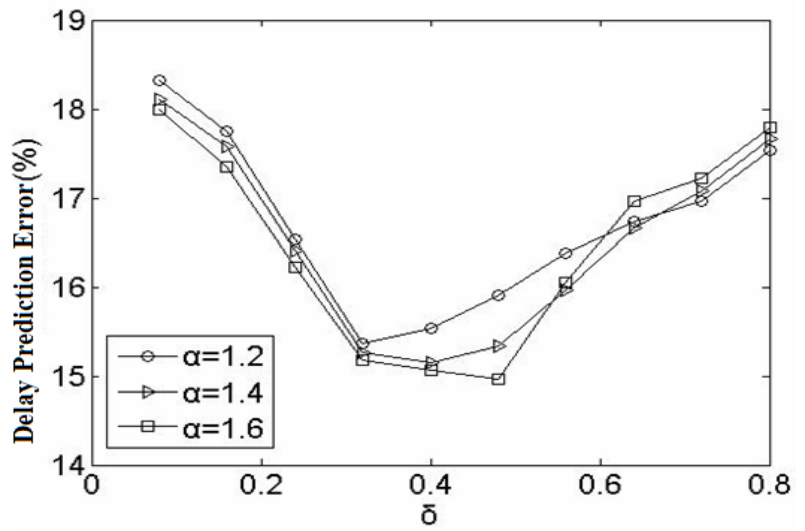

Figure 5 captain prediction error and changes in the relations between the parameters of the $\delta$

\section{Conclusion}

According to actual wireless sensor network traffic prediction accuracy problem, based on ARMA model and wavelet transform combined with a new prediction algorithm is proposed. First of all, defines the ARMA model, the algorithm. At the same time, combined with the ARMA model and wavelet transform actual traffic prediction method is established, through the integration of the two methods to predict the results to achieve the purpose of reduce the prediction error. Finally, OPNET and MATLAB joint simulation, in-depth study of the key factors that affect the algorithm, and compare the FARIMA model performance, the results showed that this algorithm has good adaptability. In a follow-up study, can consider to combine the chaos and other prediction method to establish a complete set of wireless sensor network (WSN) prediction and evaluation model. 


\section{References}

[1] Liao H, Lee C, Kedia S P. Mobile anchor positioning for wireless sensor networks[J]. IET Communications, 2011, 5(7): 914-921.

[2] Yick J, Mukherjee B, Ghosald. Wireless sensor network survey[J]. IEEE Computer Networks, 2008, 52(12): 2292-2330.

[3] Wu X B, Chen G, Das S K. Avoiding energy holes in wireless sensor networks with nonuniform node distribution[J]. IEEE Transactions on Parallel and Distributed Systems, 2008, 19(5): 710-720.

[4] Chang Y C, Lin Z S, Chen J L. Cluster based self organization management protocols for wireless sensor networks[J]. IEEE Transactions on Consumer Electronics, 2006, 52(1): 75-80.

[5] Luo X, Dong M, Huang Y. On Distributed fault tolerant detection in wireless sensor networks[J]. IEEE Transactions on Computers, 2006, 55(1): 58-70.

[6] Patwari N,Hero A.Using proximity and quantized RSS for sensor localization in wireless net works[C]//Proc of the 2nd ACM Int workshop on wireless Sensor Networks and Applications. New York:ACM, 2003:20-29

[7] Li X,Shi H C,Shang Y. A sorted RSSI quantization based algorithm for sensor network localization[C]//Pro of the 11th Int Conf on parallel and Distributed Systems. Los Alamitos,CA:IEEE Computer Society. 2005:557- 563.

[8] Shan wei,Based on nonlinear time series prediction model testing and optimization research [J]. Journal of electronics, 2008, 36 (12) : 2485-2489.

[9] Song Yang, TuXiaoMin. Internet time delay prediction based on FARIMA model [J]. Journal of instruments and meters, 2012, 33 (4) : 757-763.

[10] WenXiangXi, xiang-ru meng. Small time scale network traffic chaos analysis and trend prediction [J]. Journal of electronics, 2012, 40 (8) : 1609-1616.

[11] li dandan, run-tong zhang. Cognitive network in network traffic prediction model based on ant colony algorithm [J]. Journal of electronics, 2011, 39 (10) : 2245-2250.

[12]Li chao, zhao hai, ge new, zhang jun. Based on the chaotic characteristics of the network delay prediction model [J]. Journal of electronics, 2009, 37 (12) : 2657-2661.

[13] Lei Ting , Yu Zhengwei. A wavelet neural network model of network traffic prediction [J]. Journal of computer applications, 2006, 26 (3) : 526-528.

[14] Ye MeiYing, Wang XiaoDong,Zhang HaoRan. Based on on-line least squares support vector machine (SVM) regression of chaotic time series prediction [J]. Journal of physics, 2005, 54 (6) : 2568-2573.

[15] Zhou Manzhen, Guo Yan, liu xiaoming, Su Zhaolong. Based on the batch queue self-similar business performance analysis [J]. Journal of system simulation, 2004, 16 (2) : 306-309.

[16]N Sadek, A Khotanzad, T Chen. ATM dynamic bandwidth allocation using FARIMA prediction model[C]. In Proceedings of International Conference on Computer Communications and Networks, 2003: 359-363 